\title{
Local Variable Name
}

National Cancer Institute

\section{Source}

National Cancer Institute. Local Variable Name. NCI Thesaurus. Code C95340.

A label that represents the value of an observed result and serves as an input parameter used in the substance administration dose expression. 